\title{
Test of Tourist Risk Perception Scale after Natural Disaster: Based on Kangding Earthquake \\ Yue $\mathrm{Li}^{1, \mathrm{a}^{*}}$, Yang Yang ${ }^{2, \mathrm{~b}}$, Yawen $\mathrm{He}^{3, \mathrm{c}}$ and Qizhi Yang ${ }^{4, \mathrm{~d}}$
}

\author{
${ }^{1}$ Hotel management Department, Tourism school, Sichuan Agricultural University, Chengdu, Sichuan, \\ China \\ ${ }^{2}$ Tourism school, Sichuan University, Chengdu, Sichuan, China \\ ${ }^{3}$ Business school, Sichuan University, Chengdu, Sichuan, China \\ ${ }^{4}$ Tourism school, Sichuan Agricultural University, Chengdu, Sichuan, China \\ a26005476@qq.com, b371687937@qq.coml, ccoolerthanme4444@qq.com, ${ }^{\mathrm{d}} 714547454 @ q q . c o m$ \\ *The Corresponding author
}

Keywords: Scale; Tourist risk perception; Natural disaster; Kangding earthquake; CFA

\begin{abstract}
This study aimed to test the dimensionality of tourist risk perception under the scenario of natural disaster. Kangding earthquake affected tourism attraction was selected to test the risk perception of potential risk. Empirical study on Kangding tourism attraction after earthquake showed the tourists risk perception towards nature disaster. Eight factors were extracted from the items that measured tourists risk perception employing the exploratory factor analysis in terms of social risk, health risk, facility risk, criminal risk, ethical risk, psychological risk, financial risk and satisfaction risk. After the two-step confirmatory factor analyses by LISERL 8.7, the results of first-order CFA and second-order CFA showed the good fitness of risk perception model dimension model were acceptable. Meanwhile, measurement model demonstrated adequate reliability, convergent validity and discriminant validity. The least and most important dimensions of perceived risk towards natural disasters-satisfactions risk, facility risk, psychological risk criminal risk are ranked as the most important dimensions for risk perception; ethical and social risk are ranked as least important factors. The result provides some comprehensive management suggestions on the tourism attraction recovery. Based on the factor analysis, K-means analysis was conducted in order to distinguish four different groups of tourists on travel intention.
\end{abstract}

\section{Introduction}

Tourism as the largest industry all over the world, it has become a major component of the economic prosperity of almost all countries [1]. Tourism industry could increase the level of income and employment as well as booming the related industries [2]. Meanwhile, numbers factors can influence this industry, such as political, economic environmental factors, that reveals the vulnerability and fragility of tourism industry. Therefore, uncertainty events and crisis can destroy the whole tourism system, especially the nature disasters, economic recession, epidemic disease, terrorism attack and political unrest. Take 2008 Wenchuan earthquake as an example, hotel room hotel occupancy rate of Sichuan province decreased from $53 \%$ to $44 \%$. The number of international tourists decreased from 95,000 to 26,000 , domestic tourists declined from 1.4 million to 1.2 million. For the Taiwan 9.21 earthquake, the international tourists declined $15 \%$ and 230 tourists' attractions lost $27 \%$ tourists after the earthquake [3]. The tourists' defection problem caused by tsunami became the nightmare for the tourism industry in Indian Ocean region [4].

Risk and tourism are intrinsically related, as the decision to travel itself implicates risk, including the risk of travelling to an unfamiliar place; the uncertainty of future conditions; and the possible negative 
outcomes of making any travel-related decisions [5]. Previous investigations have identified several major risk factors: terrorism [6-8]; war and political instability[9-11]; health concerns [12-14]; and crime [15-18]; "A crisis is any unexpected event that affects traveler confidence in a destination and interferes with the ability to continue operating normally" [19]. Academic research were mainly focused on how the tourism crisis influences on tourism industry and the tourism management strategy, understanding the tourism crisis from tourists perspective was overlooked in both academic and practice. The tourism value chain theory claimed that, the fundamental purpose of the tourism industry is satisfying the consumer needs wants and demand, since the tourists plays a critical role in marketing and whole tourism value chain [20]. Therefore, understanding the tourism crisis from tourist perspective is valuable and that could support crisis management strategies to help retain the confidence of travelers and the travel industry, and to minimize the impact of a crisis on the destination.

The main features of deadly natural disasters are distinctive, such as large casualties, heavily destroyed tourist attractions, highly media exposure, long duration, huge psychological shock. Compare with the ordinary nature tourism risk, it is more difficult to recovery for tourism market and normal pricing cutting strategy and safety-ensuring strategy seem not effective under this scenario. For the further reasons, physical safety factor and price factor were important content of risk perception but not cover all dimension of risk perception. The dysfunction of these formal crisis management strategies reveals the complexity of risk perception and blurry understanding of physiological mechanism of tourists. Therefore, understanding the risk perception of tourists towards the heavily destroyed tourism attractions is essential for crisis management strategy formation as well as tourism marketing recovery. Kangding or Dardo (Tibetan), is a city and the location of the seat of Garzê Tibetan Autonomous Prefecture in western Sichuan province of Southwest China. Kangding's urban center has around 100,000 inhabitants. There was an earthquake swarm of two major events of MS6.3 and MS5.8 on the Xianshuihe fault in November, 2014.

Tourists risk perception has been widely researched in tourism studies. Whereas previous investigations on tourist risk perception scale might not have good external validity, due to the dramatic influences on perception and cognition of tourists caused by the severely destructive disaster, such as earthquake.

The extensive literature review were conducted by Yue Li [21] to provide several factors risk perception: physical risk, health risk, financial risk, social risk, time risk, facility risk, satisfaction risk and physiological risk. Ethic risk was also taken into account under the scenario of disaster. Then Yue Li [21] developed the post-disaster Tourists risk perception scale based on the data of Wenchuan earthquake in 2008.

In consequence, it is necessary to test the dimension of the tourist risk perception on disaster under different situation. Then, it can be useful on the following aspects. firstly, to understanding the psychological mechanism of tourists after a tourism attraction suffering the severely destructive disaster by measuring the dimension of tourists' risk perception. Secondly, provide a comprehensive and constructive suggestion for the government and business practitioner to improve the travelling willingness and recover the tourism market based on the analysis of tourists' risk perception.

\section{Materials and Methods}

Study Instrument. 37 items of tourists risk perception scale is adapted according to previous study [21] , comprised of scales measuring tourists perceived physic risk, financial risk, ethic risk, facility risk, social risk, psychological risk, crime risk, intention to visit. The respondents' demographic and social-economic characteristics were also included in the questionnaire. 37 items of risk perception scales were measured by means of five-point-Likert scale items with anchors ranging from (1) strongly disagree to (5) Strongly agree. 
A pilot study was conducted to create more compact research instrument. 200 questionnaire were collected in Dec 2014 in Sichuan University. Pilot sample is college students and 186 valid questionnaires are analyzed by SPSS. The 37 item risk perception scale was tested with reliability and factor analysis using pilot data. Results shows that, for Corrected Item -Total Correlation (CITC), 3 items below 0.4 and should be deleted to increase the Cronbach $\alpha$. A Factor analysis was conducted to extract underlying risk perception factors and reduce the item pool. 2 items were deleted due to below the judgment value 0.5. Finally, 32 items were selected for the final questionnaire.

Sampling and Procedures. The aim of this study was to investigate the dimension of tourist risk perception in the context of a great earthquake destroyed tourism attraction. The target population of this research was Chinese domestic tourists or potential tourists of Sichuan. The main reason of choosing domestic tourists was the huge amount and sustainable growth rate. Convenience sampling strategy was adopted for collect the data from domestic tourists. In Dec 2014, 100 university students were random collected by using snowball sampling and quota sampling technique. For 100 students, each of them was response for 9 potential tourists to fulfill the questionnaire nationally. Quota sampling selection was strictly in line with the Sichuan tourism bureau statistic data of tourists travelled to Sichuan from different area of China during 2013.The Ratio (\%) of tourists from different province is Sichuan 56.6, Chongqing 9.43,Yunnan 3.13,Shanxi 2.21, Guizhou 2.16,Beijing 2.08, Shanghai 2.77, Guangdong 1.93,Zhejiang1.79.1300 questionnaires were distributed in this way and 1155 were collected in the end.

Data Analysis. Data collection form questionnaire were computed using the Statistical Package for Social Sciences (SPSS) and Linear Structure Relations (LISREL) software. SPSS was adopted to generate the descriptive and inferential statistics. LISREL. A total of 1155 response was received and 309 cases were deleted due to missing values cross the entire case. Valid questionnaires reached to 846 and the valid response rate is $65.1 \%$.

Results

Respondent Profile. Respondents' demographic data were analyzed by descriptive statistics using the SPSS. Female respondents $(51.8 \%)$ slightly outnumbered the male respondents $(48.2 \%)$. Most respondents were relative young (90\% of respondents were under age of 30$)$. In terms of education $77.1 \%$ of respondents were university education and $12 \%$ were college education. Students comprised $76.1 \%$ of the sample. The second-largest occupation category was general employees accounting for $10.2 \%$ of sample. Over $71.7 \%$ of respondents earned a personal annual income less than 20,000 . For the overnight travel expenditure was mainly less than $2,000(44.2 \%)$, and $31.7 \%$ of respondents were between 2,000 to 5,000RMB.

Exploratory Factor Analysis. Factor analysis is used to extract a few important factors from a larger number of observed variables. It assumes that the factors are correlated with the larger number of observed variables. An Exploratory factor analysis (EFA) was conducted on the sample using the principal component method with VARIMAX rotation. Factor number was decided with the rule of extracted eigenvalue being greater than 1.0. The objective was to group the risk perception items and generate salient explainable. major principal components were extracted as constructs; minor principal components with eigenvalue less than 1 were ignored as noise; an item and the intended construct correlation (also known as factor loading) should be greater than 0.5 to satisfy convergent validity.

The final solution with the eight extracted factors explain $74.9 \%$ of the total variance. The KaiserMeyer-Olkin Measure (KMO) of sampling adequacy was 0.895 , which indicated a satisfactory level for the appropriateness of factor analysis. The chi-square value of Bartlett's test of sphericity was 19300, significant at the 0.001 level also indicating that the correlation among the variables was significant enough for factor analysis. $(\chi 2=19300$, d.f. $=496, \mathrm{p}<0.001)$ The Cronbach's alpha of total scale was 0.895, and Cronbach' $\mathrm{s}$ alphas for eight factors were 0.912, 0.841, 0.91, 0.939, 0.815, 0.88and 0.908 respectively, all above the generally agreed-upon lower limit of 0.7 , indicating satisfactory reliabilities for the overall scale and all the extracted factors. 
Eight factors were extracted from the items that measured tourists risk perception employing the EFA. The first factor was named social risk (eigenvalue $=9.345$, Variance $=29.20 \%$ ) and included the following: change the way my friends think of me, not accord with my self-image, not reflect my personality, change the way my family think of me and not accord with my social status. The second factor was named health risk (eigenvalue $=4.801$, variance $=15.03 \%$ ) and included the following: physical injury caused by debris flow, physical injury caused by aftershock, physical injury caused by landslide, food safety problem, water pollution and infectious diseases. The third factor was named facility risk (eigenvalue $=2.582$, variance $=8.07 \%$ ) and included the following: too much timing cost on travel preparation and plan, too much timing cost on transportation, too much unnecessary timing cost, communication inconvenience, basic life routine disorganized and transportation inconvenience. The fourth factor was named criminal risk (eigenvalue $=1.697$, variance $=5.31 \%$ ) and included the following: criminal target for theft, criminal target for robbing and the mass incidents. The fifth factor was named ethical risk (eigenvalue $=1.607$, variance $=5.02 \%$ ) and included the following: to satisfy curiosity, people will think I schadenfreude and people will think I go to the disaster area for fun and people will think I am schadenfreude. The sixth factor was named psychological risk (eigenvalue $=1.559$, variance $=4.87 \%$ ) and included the following: I felt nervous when I thought of travelling to Kangding after earthquake; I felt upset when I thought of travelling to Kangding after earthquake; I felt psychologically uncomfortable when I thought of travelling to Kangding after earthquake. The seventh factor was named financial risk (eigenvalue $=1.327$, variance $=4.14 \%$ ) and included the following: will not cost- effective, not be of good value for money and be financially burdening for me. The eighth factor was named satisfaction risk (eigenvalue $=1.068$, variance $=3.34 \%$ ) and included the following: I might be disappointed after travel to Kanding; I might make a wrong decision to travel to Kanding; can't meet my expectation. These eight factors were used as the measurement variables for tourist risk perception.

Confirmatory Factor Analysis. The eight-factor structure identified by EFA was then examined by a confirmatory factor analysis (CFA) with the 846-case validation sample by using the maximum like hood estimation method to ensure construct reliability and validity. CFA were used to investigate the factor structure of the items from risk perception scales described above. The factor was designated as latent variables, and the items loading on them were treated as observed variables designed to measure the latent variables. Two procedures were used to find risk perception in terms of first order factors and then second-order factor.

A confirmatory factor analysis via LISERL 8.7 was conducted to test the measurement Model. Numerous statistics can be used to assess the goodness of fit of a structural model. Six common modelfit measures were employed to measure the model's overall appropriateness of fit: the ratio of $\chi 2$ to degrees-of-freedom (df), goodness-of-fit index (GFI), adjusted goodness-of-fit index (AGFI), normalized fit index (NFI), comparative fit index (CFI) and root mean square error of approximation (RMSEA). The $\chi^{2}$ goodness-of-fit test evaluates the adequacy of the covariance matrix of the theorized model and compares the estimated coefficients to the observed covariance matrix. However, because the sample size may influence the value of $\chi 2$, a large sample size can make this test insufficient to adequately assess model fitness. The value of $\chi 2$ divided by the degrees of freedom (i.e. $\chi 2 /$ d.f.) so as to adjust for sample size is used to assess the model fit. Carmines and McIver (1981) propose that a $\chi 2 /$ d.f. rating of less than 5 indicates acceptable model fitness when using large samples. Other statistics, such as NFI, CFI, RMSEA and SRMR, have been used to assess model fitness. The accepted criteria for GFI, IFI, CFI and NFI are values larger than 0.9, whereas the accepted criteria for RMSEA and SRMR are values less than 0.08 .

Fig. 1 showed the standardized factor loading in first-order CFA of tourist risk perception. The factor loading of every item was between 0.6 and 0.8 . However, the R2 of item A3, A4, A4 (health risk); F20, F21, F22 (facility risk) and C11 (economic risk) were below 0.5. All the factor loadings in the model of tourist risk perception dimension exceeded 0.7, after deleted those items. All of the 
model-fit indices exceeded the respective common acceptance levels suggested by previous research, thus demonstrating a fairly good fit of the measurement model with the data collected $(\mathrm{X} 2 / \mathrm{df}=2.67$, RMSEA $=0.042, \mathrm{GFI}=0.94, \mathrm{SRMR}=0.031$, NFI $=0.98, \mathrm{NNFI}=0.98, \mathrm{CFI}=0.99$, AGFI=0.92). Thus, the underlying motivation structure identified by EFA was generally confirmed with the validation sample.

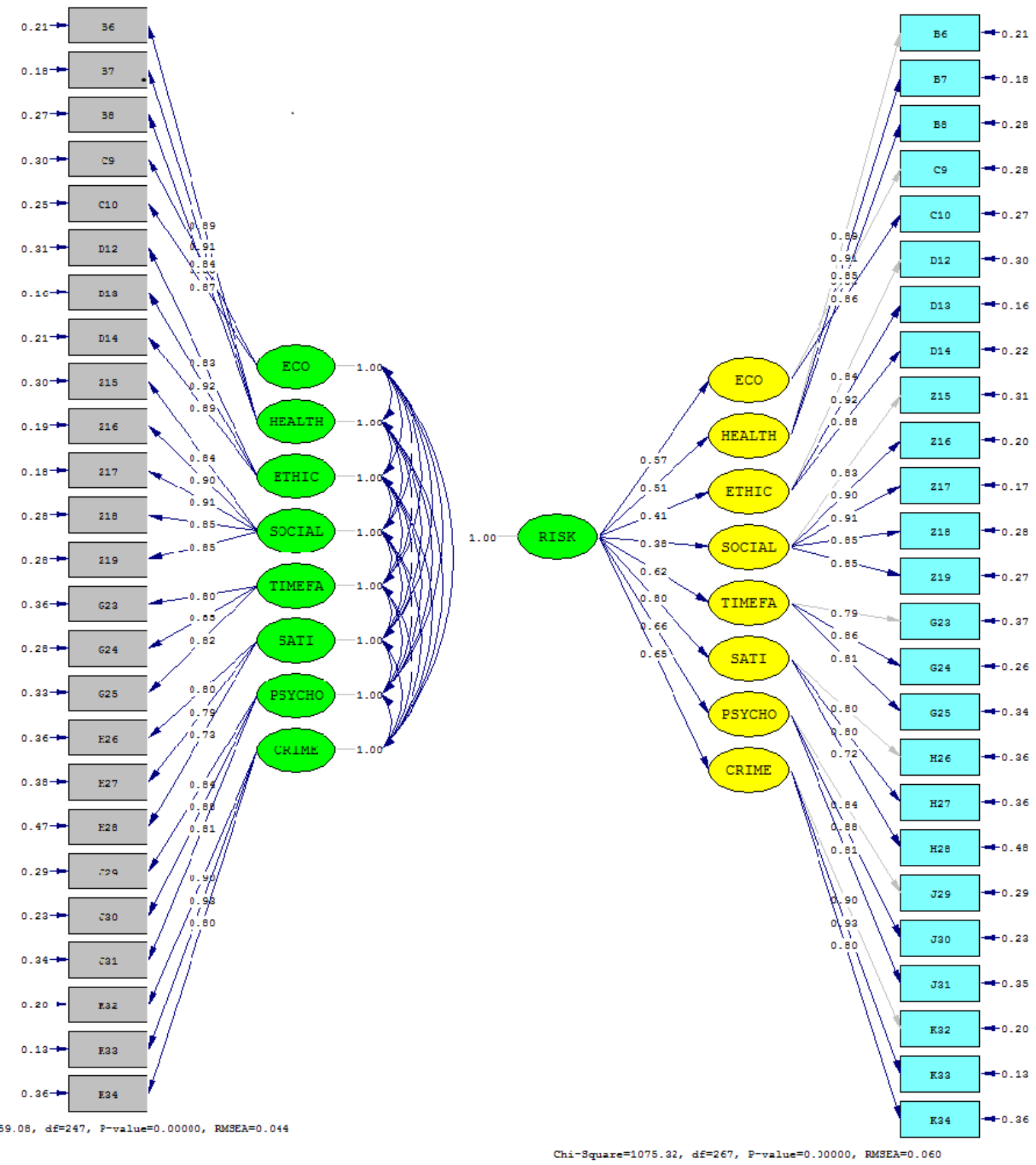

Figure 1. Finite The results of first-order CFA Figure 2. Finite The results of second-order CFA

Fig. 2 demonstrated the standardized factor loading in second-order CFA of tourist risk perception. The factor loadings of ethical risk (0.41) and social risk (0.38) were below 0.6 , the factor loading of health risk, facility risk, psychological risk, satisfaction risk and criminal risk were between 0.6 and 0.8 . 
For the model fitness, all the indicators of second-order CFA presented an acceptable fit between the CFA and the observed data $(\mathrm{X} 2 / \mathrm{df}=4.03, \mathrm{RMSEA}=0.060, \mathrm{GFI}=0.97, \mathrm{SRMR}=0.095, \mathrm{NFI}=0.96$, $\mathrm{NNFI}=0.97, \mathrm{CFI}=0.97$, AGFI=0.89), except that the AGFI was 0.89 slightly below 0.9 . The study therefore proceeded to evaluate the psychometric properties of the measurement model, namely, its reliability, convergent validity and discriminant validity.

Reliability and Validity. The composite reliability values exceeded 0.6 , showing that the latent variables had high internal consistency. The average variance extracted can be used to assess convergent and discriminant validity and should exceed 0.5 . Validity reflects the extent to which an item actually what it is supposed to measure. Validity of indicator variables can be assessed by examining the magnitude and significance of the loadings between each latent variable and its indicators. An absolute value of the $t$ statistic over 1.96 for an item's loading provides evidence of validity in favor of the item used as an indicator of latent construct.

To test discriminant validity, this study compared the shared variance between factors with the average variance extracted from individual factors. This analysis showed that the shared variances between factors were lower than the average variance extracted from the individual factors, thus confirming discriminant validity in brief, the measurement model demonstrated adequate reliability, convergent validity and discriminant validity.

K-Means Analysis. To identify the effect of different factors on tourist's travel intention, in order to distinguish different groups of tourists, clustering algorithms are generally used as the primary methodology for serving the purpose. Clustering analysis techniques have been discussed in the extensive literature. The most popular is k-means algorithm which was broadly reviewed by different authors. It is also found that algorithms using computational intelligence did not show better results than k-means, the combinations of several algorithms are very often recommended as the conclusion. The study of Zakrwska and Murlewski showed that k-means is very efficient for large multidimensional data sets, however depends strongly on the choice of input parameter $\mathrm{k}$. However, it is not recommended in the case of data sets with noise. The based in cluster analysis of eight factor scores for perceived risks, the whole sample was classified into four groups: Group 1 (group of being risk sensitive), group 2 (group of being pragmatism), group 3 (group of being self-interests priority) and Group 4 (group of being ethic social Insensitivity). ANOVA was conducted and the results show that there are significant differences among four groups on tourism intention. First of all, three subdimension of travel intention was tested by factor analysis. The results showed KMO of sampling adequacy was 0.676 , which indicated a satisfactory level for the appropriateness of factor analysis. The chi-square value of Bartlett's test of Sphericity was 739.31, significant at the 0.001 level also indicating that the correlation among the variables was significant enough for factor analysis. The result of ANOVA shows the travel intention of Group 4 is higher than Group 1. $(F=2.508, p<0.1)$

\section{Discussion}

Empirical study on Kangding tourism attraction after earthquake showed the tourists risk perception towards severe nature disaster. Eight factors were extracted from the items that measured tourists risk perception employing the exploratory factor analysis, in terms of social risk, health risk, facility risk, criminal risk, ethical risk, psychological risk, financial risk and satisfaction risk. After the two-step confirmatory factor analyses by LISERL 8.7, the results of first-order CFA and second-order CFA showed the good fitness of risk perception model dimension model were acceptable.

The least and most important dimensions of risk perception, as held by domestic tourists, are identified in this study. Each of the sub-dimensions varies in terms of their importance to the eight primary dimensions. Satisfactions risk is confirmed to be the most important dimension of risk perception towards severe natural disasters. In this study, satisfactions risk is confirmed to be the most important dimension of risk perception towards severe natural disasters. In this study, Facility risk is 
composed of three significant sub-dimensions, namely disappointment $(\mathrm{b}=0.80)$, wrong decision $(\mathrm{b}=$ $0.80)$ and not meet the expectation $(b=0.72)$. Psychological risk is confirmed to be the second most important dimension of risk perception towards severe natural disasters. In this study, psychological risk comprises three significant sub-dimension, namely, nervous $(b=0.84)$, upset $(b=0.88)$ and psychologically uncomfortable $(b=0.81)$. The result of - this study agrees with pervious study. Criminal risk is perceived as the third most important dimension of risk perception. The finding support the contention of Karagiannis's study(15) that criminal risks is perceived as the most important dimension when tourists make their overall assessment of perceived risk. In this study, criminal risk is composed of three important sub-dimensions, namely, criminal target for theft $(b=0.90)$ criminal target for robbing $(b=0.93)$ worry about personal safety $(b=0.80)$. In this study, Satisfactions risk as the fourth significant dimensions is composed of three significant sub-dimensions, namely communication inconvenience $(b=0.79)$, infrastructure inconvenience $(b=0.86)$ and transportation inconvenience $(b=0.81)$. On the contrary, in this study, social risk $(b=0.41)$ and ethic risk $(b=0.38)$ rated as two least significant dimension of perceived risk towards severe natural disasters. The reason for this phenomenon could be the propaganda of government to deliver a message of tourism could helpful for disaster area recovery. Therefore, travelling to Kangding do not case many ethical and social pressure. Compare with these two factors, economic risk and health risk still play important role on risk perception in this study. The result provides some comprehensive management suggestions on the tourism attraction recovery. Meanwhile, measurement model demonstrated adequate reliability, convergent validity and discriminant validity. For the future research, tourist risk perception dimension could be taken as an independent variable to explore the relations with tourists travel intention by adopting LISERL and AMOS. More work should be done to explore the mechanism of tourists' decision and constrain. For the government and business practicer, under the scenario of severe disaster like Kangding earthquake, management strategies should be taking tourists' ethical and psychological risk in to account, rather than the normal crisis management strategy which mainly focused on financial risk and physical risk. Meanwhile provide the comprehensive theoretical foundation to help retain the confidence of travelers and the travel industry, and to minimize the impact of a crisis on the destination. Besides, during the different stage of crisis management, the tourists risk perception should be adjusted to fit the scenario of different requirements.

\section{Conclusion}

The tourist risk perception dimension towards the severe nature disaster is constituted with 32 items eight dimensions, in terms of social risk, health risk, facility risk, criminal risk, ethical risk, psychological risk, financial risk and satisfaction risk. This scale can provide a reliable measurement of risk perception due to its reliability and convergent validity as well as the good model fitness. Meanwhile, this study identifies the importance of these eight factors of risk perception. satisfactions risk, facility risk, psychological risk and criminal risk are ranked as the most important dimensions for risk perception; ethical and social risk are ranked as least important factors. The result provides some comprehensive management suggestions on the tourism attraction recovery. Based on the factor analysis, K-means analysis was conducted in order to distinguish four different groups of tourists on travel intention. Besides, the results of second- factor analysis showed the good fitness, which means it could be simplified for the future model relationship analysis. From the theoretical perspective, understanding the dimension of tourist risk perception is the cornerstone of tourists' behavior under the scenario of severe nature disaster. The relationship among risk perception, destination image, destination familiarity and tourists travel intention could reveal the tourists' psychological mechanism when he or she traveled to the disaster area. Meanwhile understanding the tourists risk perception is helpful for rebuilding the destination image as well as tourists market recovery. Moreover, helping 
retain the confidence of travelers and the travel industry, and to minimize the impact of a crisis on the destination.

\section{Acknowledgments}

Supported by Young Expert Training Program in Tourism Industry, Sichuan Tourism Development and Reform Commission (SCTYETP2016L32); by Scientific Research Foundation for Returned Scholars, Ministry of Human Resources and Social Security of China (to Li Yue); by Project of Education Department in Sichuan(15SB0322).

\section{References}

[1] Goeldner CR, Ritchie JRB, Goeldner CR, Ritchie JRB. Tourism : principles, practices, philosophies: John Wiley; 2006.

[2] Kandampully $\mathrm{J}$. The impact of demand fluctuation on the quality of service: a tourism industry example. Managing Service Quality. 2000; volume 10(1):10-9.

[3] Huang JH, Min JCH. Earthquake devastation and recovery in tourism: the Taiwan case. Tourism Management. 2002;23(2):145-54.

[4] Huang JH, Chuang ST, Lin YR. Folk Religion and Tourist Intention: Avoiding Tsunami-Affected Destinations. Annals of Tourism Research. 2008;35(4):1074-8.

[5] Chang SY. Australians' holiday decisions in China: a study combining novelty-seeking and riskperception behaviors. Journal of China Tourism Research. 2009;5(4):364-87.

[6] Sonmez SF, Apostolopoulos Y, Tarlow P. Tourism in Crisis: Managing the Effects on Tourism. Journal of Travel Research. 1999;38.

[7] Aziza H. Understanding attacks on tourists in Egypt. Tourism Management. 1995;16(2):91-5.

[8] Leslie D, Pizam A, Tarlow P. Terrorism and Tourism: The Northern Ireland Situation-A Look behind the Veil of Certainty. Journal of Travel Research. 1999;38:37-40.

[9] Page SJ, Lawton GR. Tourism, crime and international security issues: Edited by A. Pizam and Y. Mansfeld. Wiley (Baffins Lane, Chichester PO19 1UD, UK), 1996, viii pp. 321 . Annals of Tourism Research. 1997;24(2):487-9.

[10] Poirier RA. Political risk analysis and tourism. Annals of Tourism Research. 1997;24(97):675-86.

[11] Piekarz MJ. Tourism in an unstable and complex world? : searching for a relevant political risk paradigm and model for tourism organisations. Cranfield University. 2008.

[12] Wickens E. Health risk-taking and tourism. Asean Journal on Hospitality \& Tourism. 2003:160-70.

[13]Peattie S, Clarke P, Peattie K. Risk and responsibility in tourism: promoting sun-safety. Tourism Management. 2005;26(3):399-408.

[14] Sánchez-Quiles D, Tovar-Sánchez A. Are sunscreens a new environmental risk associated with coastal tourism? Environment International. 2015;83:158-70.

[15] Karagiannis N, Madjd-Sadjadi Z. Crime, criminal activity and tourism performance: issues from the Caribbean. Worldwide Hospitality \& Tourism Themes. 2012;4(1):73-90. 
[16]Barker M, Page SJ, Meyer D. Modeling tourism crime: the 2000 America's Cup. Annals of Tourism Research. 2002;29(01):762-82.

[17] Barker M, Page SJ, Meyer D. Modeling tourism crime. Annals of Tourism Research. 2002;29:76282.

[18]Tynon JF, Chavez DJ. Adapting a Tourism Crime Typology: Classifying Outdoor Recreation Crime. Journal of Travel Research. 2006;44:298-307.

[19] WTO. 2003, Crisis Guideline for the Tourism Industry. 2003.

[20] Georgantzas NC. Tourism dynamics: Cyprus' hotel value chain and portability. System Dynamics Review. 2003;19(3):175-212.

[21] Yue Li, Wei Li. Study on Post-disaster Tourist Risk Perception Scale Based on Wenchuan Earthquake. Journal of Southwest University for Nationalities. 2013;10(34):21-25. (In Chinese) 